Review Articles

\title{
Methods of Assessing Diabetic Control
}

\author{
G. D. Molnar, G. J. Marien, A. N. Hunter, and C. H. Harley \\ Department of Medicine, University of Alberta, Edmonton, Alberta, Canada
}

\begin{abstract}
Summary. Control of diabetes from complete normalisation to less adequate degrees of metabolic regulation needs to be assessed with regard to conditions of evaluation and to severity of the disease. Under optimal conditions the therapeutic events should occur with well-timed regularity. Different assessment criteria are appropriate depending on the severity of the deficiency of endogenous insulin. Plasma and urine glucose and ketone body measurements remain the practical standards for assessing diabetic control. Abnormalities of lipid and protein metabolites serve to augment the scope of the assessment. Triglycerides and haemoglobin $\mathrm{Al}_{\mathrm{c}}$ are also useful indicators of control. In mild (Type II) diabetes it may be possible to achieve normal plasma glucose measurements two hours after meals. Such aims carry a risk of hypoglycaemia in severe (Type I) diabetes. Normoglycaemia and aglycosuria in severe diabetes are feasible only preprandially in most cases. The use of urine glucose tests requires evaluation of blood-tourine glucose relationships. Practical and convenient methods for identifying patients with high or low "renal thresholds" are described. Investigational methods for characterising diabetic patients assess the variability of glucose and other variables during therapy, as well as the degree to which normal values are attained. Such assessment methods may gain increasing practical importance as therapeutic approaches to diabetic control which are experimental at present come into practice.
\end{abstract}

Key words: Diabetic control, $\mathrm{H}_{\mathrm{b}} \mathrm{AI}_{\mathrm{c}}$, hyperglycaemia, diabetes, renal threshold.

"Control" of Diabetes Mellitus is a much misunderstood phrase. It implies the act of controlling, regulating or restraining the metabolic disturbances engendered by the lack of insulin. That there are degrees of control is obvious, but this is not always recognized. A totally insulin-deficient diabetic patient is controlled, when an artificial endocrine pancreas temporarily restores the moment-tomoment normality of blood glucose concentration [1]. The same patient, however, may also be described as "controlled", when minimal dietary and insulin therapy merely prevents the development of ketoacidosis. The former degree of control is ideal with regard to blood glucose, while the latter is barely adequate clinically.

Frustration with the concept of control of diabetes is compounded by disagreements about criteria of control. On what measurements should the assessment of control be based? Are measurements of glucose in blood and urine sufficient, given that the effects of insulin are known to affect many different metabolic pathways? Even if restricted to measurements of glucose in blood, what values (and by which method of analysis) are to be used for defining perfect, adequate and poor control of diabetes? Even if the ranges of plasma glucose concentration by an universally acceptable method were to be acceptable as defining these degrees of control, under what conditions are blood samples for these measurements to be obtained? For instance, how many hours after the ingestion of what nutrients should postprandial plasma glucose measurements be made?

The concept of diabetic control is further complicated by the need to recognise the variability of results on re-testing the same patient, even under identical conditions. When such variability is low or moderate, diabetologists speak of stable diabetes. Highly variable results during consistent conditions of testing or therapy justify the designation of labile diabetes. It is true that patients whose glycaemia is nearest to normal, tend to be least variable and Patients whose blood glucose levels are farthest from 
normal tend to be most variable, although there are many exceptions. Thus a labile diabetic patient may be only moderately hyperglycaemic, but highly variable from day to day. A stable diabetic patient may be more severely hyperglycaemic, but much less variable from day to day [2].

This review focuses on what should be measured, when, under what conditions and with what results. We begin by examining the conditions under which the assessment occurs and what bearing the severity of the diabetes has on the control that is achievable.

\section{Conditions for Evaluation}

Conditions generally necessary for evaluation of diabetic control are consistency of therapeutic events, with accurate timing and realistic scheduling so as to yield decisive, consistent and interpretable results.

Consistent preparation of the patient is the most important factor. In diagnostic and subsequent retesting this includes dietary preparation for glucose tolerance tests. In treated patients, preparation involves day-to-day consistency of therapy with diet, insulin and exercise.

Accurate timing of the tests on blood and urine with regard to diurnal events such as food intake, insulin injections and exercise are also essential.

The first step toward achieving consistency of clinically important events is to inquire about the patient's habits with regard to meals, snacks, urine tests, insulin injections, exercise and sleep. The next step is to devise a schedule with the patient in order to make these events happen as consistenly as possible from day to day. Needless to say, unless this schedule is recorded for both the patient and the physician, it is not likely to be followed or remembered.

Distinguishing between practical and investigative conditions for evaluation of diabetic control is important, because it enables one to define criteria of control realistically.

The practical approach applies to the usual clinical situation in the office, outpatient clinic or hospital. The patient and the physician are intent on achieving the best feasible diabetic regulation. The patient may not be willing or able to keep the conditions consistent. Blood and urine sampling may also not be feasible at the precise times considered optimal. For the physician, the blood and urine test procedures available may be limited by the capabilities of available laboratories. The purpose of the assessment of diabetic control is optimisation of therapy. The goals of therapy have to be a compromise between the ideal and the feasible. The above condi- tions and the patient's safety determine the level of this compromise.

The investigational approach pertains to the assessment of diabetic control in a research setting. Adequate documentation of the diabetes is important. The completeness of documentation is crucial. The goals of investigational therapy should be normalisation of metabolism; the patient's safety can best be assured under research ward conditions.

Mild and severe diabetic patients need to be considered separately when defining realistic criteria of diabetic control.

The single most important characteristic enabling patients to achieve satisfactory regulation, is the ability to secrete and release some endogenous insulin to supply at least part of their own needs [3, 4]. Adequate regulation is usually accomplished by additional therapy. When endogenous insulin secretory ability is virtually or totally absent, it is generally not possible to attain near-normality using currently available therapy. The difference between these types of patients is due to insulin being supplied according to the body's moment-to-moment needs in the patient who retains a significant degree of insulin secretory ability, but not in the patient totally deficient of endogenous insulin [3, 4]. Accordingly, expectations for the results of therapy in these types of patients are best defined separately: close to normal values when insulin secretory reserve is adequate and more remote from normal when endogenous insulin is unavailable.

Categorising diabetic patients with adequate insulin reserve is easy, when diet therapy alone is adequate and achieves near-normal biochemical regulation or when diet and oral antidiabetic drugs are effective. In insulin-treated patients however, withdrawal of insulin therapy under close clinical scrutiny, or tests of insulin secretory reserve may be necessary to decide whether an appreciable degree of endogenous insulin reserve is present [3]. The residual reserve of insulin secretion (in insulintreated patients) is best measured by radioimmunoassay, of the connecting (C)-peptide [3, 5]. Categorising patients by their insulin secretory reserve is practically more helpful and scientifically more satisfying than by their age at onset of diabetes or by their proneness (or resistance) to ketoacidosis.

Recent observations suggest that insulin-dependent (totally insulin deficient) diabetes may be associated with histocompatibility markers (HLA types B8, BW15, DW3 and DW4) [6]. No HLA types have been found to be associated with the diabetes in which insulin-secretory reserve tends to persist, but with HLA types B7 and DW2 diminished likelihood of the individual's susceptibility to any 
kind of diabetes is suggested [6]. Histocompatibility typing may therefore be a means for assessing a patient's genetic predisposition to insulin-dependent diabetes, although it can never indicate more than a probability.

We suggest that the availability of endogenous insulin should be the basis for defining patients. Predictions may then be made in advance of the probable outcome of optimal clinical therapy. In the final analysis, consistently good therapeutic results are feasible only when the therapy assists and supports endogenous insulin supply.

\section{Clinical Evaluation of Diabetic Control}

Judging the presence and the degree of metabolic derangements by symptoms and clinical observation for appropriate signs requires skill on the part of the physician and the patient's informed awareness. Whereas the clinical manifestations are likely to be subtle, the underlying metabolic derangements are often gross before any clinical changes can be perceived. This should not deter proper observation and recording of symptoms and signs by patient, nurse or physician so that their significance can be assessed, when appropriate laboratory results become available.

The symptoms and signs suggestive of deranged diabetic control are neither pathognomonic for diabetes nor specifically indicative of the metabolic derangement to which they are clinically related. Thus polydipsia, polyphagia and weight loss occur in diseases other than diabetes. Moreover, even in diabetics they are not exclusively related to hyperglycaemia and glycosuria. These clinical findings are valuable clues to gross derangement of metabolic control. Symptoms of fatigue and irritability may also indicate poor control.

To the enlightened diabetic patient some symptoms may recall some past association with disturbed control. Nausea and abdominal pain may have been a part of previous ketoacidosis. Some patients are able to relate the disturbance of their diabetic control with variations in visual acuity or dryness of the throat. Genital pruritus and parasthaesiae generally indicate deterioration of diabetic control. Relatively more specific and somewhat more dependable are the symptoms by which patients gauge various stages and degrees of hypoglycaemia.

Among signs, weight loss, and skin infections, moniliasis, and balanitis or vulvitis are usually indicative of inadequate therapy. The pallid, tremulous and confused patient may be manifesting signs of hypoglycaemia. Hypothermia may be a manifestation of hypoglycaemia [7] and eruptive xanthomata a sign of undertreated diabetes.

Clinical manifestations for appraisal of diabetic control must usually not be accepted at face value. Laboratory confirmation must be sought. Thus, beyond attentive and observant attitudes by physicians and patients the main emphasis in assessing diabetic control must clearly rest on laboratory methods and their interpretation.

\section{Laboratory Methods of Assessing Control of Diabetes}

The widespread effects of insulin on metabolism of carbohydrate, fat and protein underlie the reasons for expecting that abnormalities of appropriate circulating metabolites would reflect qualitative and quantitative derangements of diabetic organisms. This expectation is borne out by observations under appropriate conditions. Accordingly, not merely glucose but also other carbohydrate, lipid and protein metabolites reflect varying degrees of insulin deficiency (and possibly other hormonal disturbances as well).

At present, glucose measurement in body fluids is still the standard whereby the clinical and biochemical status of a diabetic patient is judged. This is because of, first, the wealth of data available about glucose in body fluids in relation to the state of the diabetic organism and, second, the relative dearth of information concerning the precise relationship of the other metabolic variables to glucose on the one hand and to the less definable assessment of overall control of diabetes on the other hand.

It is not surprising therefore, that evaluation of the control of diabetes is still based mainly on glucose measurements. Few other measurements, with the exception of ketone bodies, have wide clinical use except under research conditions and it is fitting that we start with a consideration of glucose measurements.

\section{Carbohydrate Metabolism - Glucose in Blood and Urine}

Many methods for analysis of glucose exist [8]. Plasma glucose measurements as representative of extracellular fluid concentration are increasingly preferred over whole blood glucose. Semiquantitative urine glucose measurements are widely used clinically, with quantitative methods being usually reserved for investigational use. Glucose in some other tissue fluids (tears [9] and cerebrospinal fluid) has been shown to have appropriate correlations with 
Table 1. Diabetic control. Blood to urine glucose relationships

$\begin{array}{ll}\text { Estimation } & \begin{array}{l}\text { Urinalyses for glucose immediately before } \\ \text { after each blood sampling for glucose analysis } \\ \text { ("bracketing") }\end{array} \\ \text { "Threshold" } & \begin{array}{l}\text { May be high if no glycosuria } \\ \text { with plasma glucose } \geq 200 \mathrm{mg} / \mathrm{dl}\end{array} \\ & \begin{array}{l}\text { May be low if glycosuria } \\ \text { with plasma glucose } \leq 150 \mathrm{mg} / \mathrm{dl}\end{array} \\ \text { Confirmation needed under better defined conditions }\end{array}$

a Bladder having been emptied within the previous hour

Table 2. Diabetic control. Clinical confirmation of abnormal blood to urine glucose relationships

\begin{tabular}{cl}
\hline Principle & $\begin{array}{l}\text { Simultaneous hourly blood and urine glucose } \\
\text { determinations under conditions to ensure } \\
\text { plasma glucose concentration in range of sus- } \\
\text { pected abnormality }\end{array}$ \\
Procedure & \\
Preparation & $\begin{array}{l}\text { Previous "bracketing" } \\
\text { Rule out uropathy }\end{array}$ \\
Sampling & $\begin{array}{l}\text { Blood and urine for glucose analysis hourly } \\
\text { from } 1100 \mathrm{~h}\end{array}$ \\
Conditions & $\begin{array}{l}\text { Water, } 200 \text { ml each hour, to enhance urine flow } \\
\text { lunch at } 1200 \mathrm{~h}\end{array}$ \\
& $\begin{array}{l}\text { Additional insulin" or additional food or } \\
\text { glucose }\end{array}$ \\
Follow-up & $\begin{array}{l}\text { Investigate aetiology if abnormality confirmed } \\
\text { with high "threshold": } \\
\text { test early after meals with highly sensitive } \\
\text { reagent (aim for negative results) } \\
\text { with low "threshold": } \\
\text { test late after meals with less sensitive rea- } \\
\text { gent (aim for lowest acceptable degrees of } \\
\text { glycosuria) }\end{array}$ \\
Indoctrinate if capillary glucose self-testing \\
recommended
\end{tabular}

a Before lunch; if needed to ensure normoglycaemia late-postprandially

b With lunch; if needed to ensure high enough glycaemia postprandially

blood glucose levels, but has not been found to have practical clinical value.

Quantitative analysis in well-timed urine collections provides one of the best indicators of diabetic control, being highly correlated with the mean of simultaneously recorded continuously-analysed blood glucose [10]. Semiquantitative urine glucose analyses, however, have many problems. This is especially so with casually collected urine specimens, which, although convenient often yield results which can be misleading with reference to the prevailing glycaemia at the time of voiding [10]. The reasons for providing misleading information are numerous. These include faulty urine collection, faulty test equipment, poor test technique and inadequacies of the test being used. There may also be problems in individual patients concerning their blood-to-urine glucose relationships ("renal threshold") and rapid changes of blood glucose concentration at (or near) the time of urine collection $[10,11]$. These inadequacies of clinical urine glucose testing pose major problems for patients and physicians alike.

A number of recent studies of semiquantitative urine glucose analyses attest to the fact that no one method is perfect [11].

A method of making some clinical observations about a patient's blood-to-urine glucose relationship is outlined in Table 1. By having the patient test a specimen of urine for glucose content immediately before and after each plasma glucose determination ("bracketing"), a rough estimate of the patient's "renal threshold" will emerge. After a few days of such tests an impression of "appropriate" of "inappropriate" blood-to-urine glucose relationship is likely to appear. If there is repeatedly glycosuria in the urine specimens, which preceded or followed the blood sampling, at plasma concentrations of $150 \mathrm{mg} /$ dl or less a "low renal threshold" may be suspected. With no glycosuria in urine specimens, which preceded (or followed) the blood sampling, at plasma concentrations of 200 (or even 300) mg/dl a "high renal threshold" may be suspected.

A more clearly documented clinical estimate of the "renal threshold" can be obtained as follows (Table 2). An afternoon is dedicated to the task, under conditions which ensure that the patient's glycaemia will encompass the range of concentrations about which information is being sought. Thus, if a low threshold is suspected some additional soluble insulin may be given before lunch, to make it more likely that late post-prandial euglycaemia or mild hypoglycaemia will occur. If a high renal threshold is suspected the lunch may be "reinforced" with a suitable glucose drink, to produce hyperglycaemia of appropriate magnitude. The mid-afternoon snack is usually omitted, but otherwise the patient should be on his usual therapeutic regimen. From an hour before lunch until the evening meal blood sampling and urine collections are made hourly. Sufficient water intake is provided by hourly drinks. The urine is analysed both by quantitative and semiquantitative methods. Some hyperglycaemia after lunch and lower glycaemia before the next meal should provide the setting against which the clinical aspects of the "renal threshold" are explored (Table 2).

For the patient with variable blood-to-urine glucose relationships, rapid changes of circulating glu- 
cose levels need to be ascertained [10]. This may be done by blood sampling at 15 -minute intervals for two or more hours before meals, during half-hourly urine glucose analyses done quantitatively and semiquantitatively.

The advice to the patient with a demonstrated high or low threshold needs special consideration. With a high threshold, the aim should be negative one or two hour postprandial urine tests (with a sensitive method). With a low threshold, tests done four or five hours postprandially (with a moderately sensitive method) showing an appropriate degree of glycosuria may be recommended.

Application of the information gained about blood and urine glucose interrelationships is relevant to all diabetic patients.

\section{Lipid Metabolism}

Alterations of diabetic control, as reflected in circulating lipids, become evident at different rates for three different aspects of lipid metabolism. Nonesterified fatty acid levels increase in the circulation most rapidly, and transiently, as lipolysis occurs on a time-scale of minutes. Ketone bodies respond more slowly and persist longer in the circulation. The timescale is in hours, especially for acetoacetate. Triglycerides and cholesterol respond much more slowly and less directly and dependably as diabetic control is altered, with major changes requiring weeks to occur.

The causes of changes in each of these lipids are complex and only non-esterified fatty acids and ketone bodies are closely related to diabetic control. Non-esterified fatty acid levels increase as insulin action decreases and lipolytic hormones (catecholamines, glucagon, cortisol, growth hormone, ACTH and others) exert their influence through cyclic AMP [12]. Numerous other factors are also involved, but ultimately insulin and counter-insulin effects predominate. Ketone bodies increase as nonesterified fatty acids in excess contribute to hepatic ketogenesis. 3-Hydroxybutyrate in particular accumulates in the circulation, as its peripheral uti -lisation diminishes, when insulin action decreases $[13,14]$. ACTH and cortisol are essential for diabetic ketosis to occur [15]. Plasma triglyceride and cholesterol levels become increased only after prolonged and severe insulin deficiency or inappropriate insulin action also of prolonged duration. Whereas high levels of ketone bodies in diabetics may safely be assumed to represent derangements of diabetic control, the same assumption cannot be stated with certainty for high levels of triglycerides or cholesterol. It is however, reasonable first to test the efficacy of improved metabolic control in a hyperlipaemic diabetic patient. If after some weeks the patient becomes normolipaemic, the therapeutic trial has confirmed the appropriateness of treating the lipid disorder through intensified treatment of the diabetes. It is well to keep in mind the potential of other pathogenetic mechanisms of hyperlipaemia in diabetic patients [12].

Ketone body measurements have been proven to be useful for evaluation of diabetic control and to be sensitive indices of insulin deficiency [13-15]. At lower levels of circulating insulin than those necessary to affect glucose entry into cells, metabolic clearance of ketone bodies (especially 3-hydroxybutyrate) is influenced [14]. Nevertheless, ketone body concentrations in blood are not used clinically in estimating metabolic control of diabetes. Serum ketone body determinations are usually not done until diabetic control is sufficiently disordered so that ketonuria is evident or symptomatic indications of the disturbance occur. This does not mean that serum ketone body determinations may not be useful adjuncts to the evaluation of diabetic control [16]. Such studies may disclose valuable data especially in totally insulin-deficient patients who demonstrate proneness to develop ketoacidosis at more rapid rates than average.

Urinary ketone bodies are, of course, useful and widely applied in the evaluation of diabetic control. Quantitative methods, applied to the same collections used for urine glucose quantitation, disclose useful research data [16]. The semiquantitative testing of urine for ketone bodies is of unquestioned clinical value in evaluating diabetic control. Unlike semiquantitative tests for urine glucose, the tests for ketone bodies are sensibly graded to indicate small, moderate or large amounts and not fine shadings of concentrations. Generally available products for testing measure only acetoacetate and acetone, but not 3-hydroxybutyrate. This is their greatest drawback, in view of the greater concentration of 3-hydroxybutyrate $[11,13]$.

\section{Protein Metabolism}

Although the protein anabolic effects of insulin have long been known, measurements of protein metabolism have only recently become sufficiently specific and precise to disclose, that diabetics have protein as well as glucose intolerance [17]. Measurements, appropriate for studies of nitrogen balance, disclose changes only after severe and prolonged insulin deficiency (for instance after halving an insulin-sensitive patient's daily insulin dosage) when ketosis is also evident [16]. With careful amino acid measurements however, the effects of impaired diabetic control 


\section{INSULIN INFUSION}

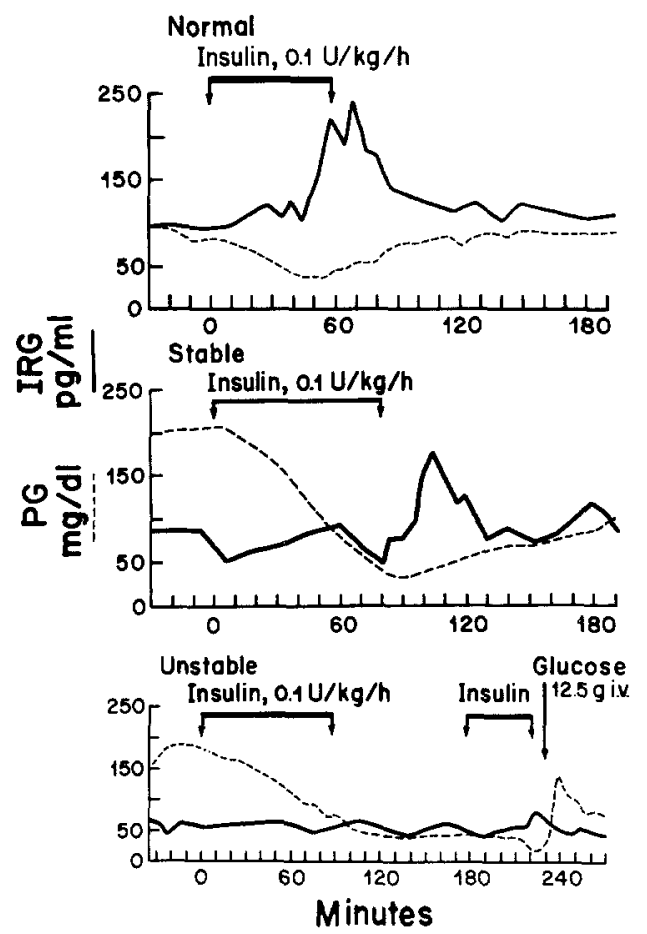

Fig. 1. Plasma immunoreactive glucagon (IRG, solid line) and glucose ( $P G$, interrupted line) during insulin infusion tests. Insulin, $0.1 \mathrm{unit} / \mathrm{kg}$ body weight $/ \mathrm{h}$ was infused. Above, a normal volunteer: middle, a stable diabetic patient (with documented insulin secretory reserve); below, an unstable diabetic patient (with documented total lack of insulin secretory reserve). Note the similar degree of hypoglycaemia in all three subjects, but much prolonged in the unstable diabetic. The glucagon response to hypoglycaemia is impaired in the stable and absent in the unstable diabetic patient [27]

soon appear. These are accelerated intracellular amino acid oxidation associated with decreased uptake of exogenous amino acids and repletion of nitrogen in muscle [17]. These changes are particularly evident with alanine, whose blood level is decreased with hyperglycaemia, while the levels of branched-chain amino acids (valine, leucine, isoleucine) are increased. Hepatic and muscle uptakes of these amino acids in the fasted and fed states and the effects of insulin provide powerful tools for investigation of this important aspect of protein metabolism in diabetes [17].

Not only nitrogen balance and plasma amino acids, but also urinary albumin (and other smaller proteins) have been shown to be related to diabetic control $[18,19]$. Although clinical tests (sulphosalicyclic acid and Albustix) may remain negative, quantitative analysis of urinary albumin shows increases in poorly controlled diabetics. With improved diabetic control the urinary albumin excre- tion may return to the normal range $[18,19]$. Albumin excretion appears to be sensitive to increased filtration pressure as well as increased filtration surface [18]. Accordingly, exercise which increases filtration pressure can be used to reveal subclinical glomerular lesions as can the infusion of dibasic amino acids, which inhibit tubular reabsorption of albumin [18].

Glycosylation of haemoglobin to an increased degree, is a further change in protein metabolism brought about by chronic hyperglycaemia. The index haemoglobin is $\mathrm{AI}_{c}$. It differs from $\mathrm{HbA}$ in having glucose and mannose on its terminal nitrogen on the B (beta) chain.

Glycosylated haemoglobin is useful as a measure of the degree of long-term glycaemic control in diabetes. The normal range for $\mathrm{Hb} \mathrm{AI}_{c}$ is $5-8 \%$ of the total haemoglobin concentration. In ketoacidotic patients the proportion is doubled to $16 \%$ or more [21]. By controlling diabetes to the clinically satisfactory level, which conventional (but non-normalising) therapy provides, the proportion of glycosylated haemoglobin is decreased to $10-14 \%$ [20-22]. $\mathrm{Hb}$ $\mathrm{AI}_{\mathrm{c}}$ concentration is highly correlated with the overall clinical assessment of diabetic control and with the mean blood glucose and fasting glucose concentration but apparently not with immunoreactive insulin concentration [23]. According to a recent study, for a given change in fasting plasma glucose, the corresponding change in $\mathrm{Hb} \mathrm{AI}_{\mathrm{c}}$ is greater as normoglycemia is approached [23].

As with triglycerides, $\mathrm{Hb} \mathrm{AI}_{\mathrm{c}}$ changes reflect changes in mean blood glucose concentration of several weeks' duration. Moreover, this post-synthetic glycosylation is detectable only as new erythrocytes form, so that about one month is needed for detection of changes [20, 21]. Indeed, $\mathrm{Hb} \mathrm{AI}_{\mathrm{c}}$ and triglyceride changes are highly correlated [22]. Pending information to the contrary, these two measures appear suited for the evaluation of long-term control of glycaemia. The subject has been revieved recently in detail in this journal [24].

\section{Hormones}

Although the principal use of insulin assay in evaluation of metabolic control is investigative, an appropriate clinical application of insulin assays is their use for categorisation of patients on the basis of insulin secretory reserve. C-peptide assay must be done if the patient has been treated with insulin and if endogenous insulin-production is to be measured.

In addition to measurements of immunoreactive insulin (IRI) and C-peptide in blood, measurements of urinary insulin are also feasible $[25,26]$, and may 
be more useful than serum IRI when integrated values over extended periods of time are desired. Conditions for the urinary C-peptide assay are more favorable than for IRI. About $5 \%$ of C-peptide released from the islets appears in the urine, but only $0.1 \%$ of IRI [26]. Measuring the endogenously secreted insulin in urine is therefore a useful approach to the evaluation of special aspects of diabetic control. It makes possible comparisons of patients with themselves under various conditions such as infections or experimental stresses and permits studies during renal disease [25].

Other hormonal measurements such as glucagon, cortisol, thyroxine, growth hormone, somatostatin and others, have at present, mainly investigational uses in the evaluation of diabetic control. It is well recognized, however, that documentation of a coexisting adrenocortical or pituitary dysfunction has important clinical relevance to the therapy of diabetes. It is less well appreciated, that a patient's ability to release glucagon in response to hypoglycaemia may also be clinically important and therefore be worth testing [27]. The lack of glucagon increase during hypoglycaemia is a characteristic of some totally insulin deficient "brittle" diabetic patients (Figure 1). Although there is no replacement therapy for this defect in patients, who when euglycaemic and hyperglycaemic have normal or high immunoreactive glucagon levels, awareness of their lack of defense against hypoglycaemia is nonetheless clinically useful [27].

Insulin antibody measurements are of limited clinical usefulness in relation to evaluation of diabetic control. High levels of insulin-binding antibodies in insulin-resistant patients have therapeutic relevance [28], however, the claim of low levels being a cause of brittle diabetes [29] is controversial [30].

Insulin receptors on the surface of target cells are regulators, as well as initiators, of target cell response [31]. By measuring both receptor concentration and affinity, both normal and pathological variations in insulin responsiveness should become more understandable, but data are lacking on the use of these measurements in the evaluation of diabetic control.

\section{Assessing Diabetic Control}

\section{Mild Diabetes}

Practical Approach. In the mildest diabetic, whose diagnosis may have required an oral (or intravenous) glucose tolerance test, repetition of the same test may be a suitable assessment after a period of therapy. The benefit of weight reduction, when this is neces-
Table 3. Diabetic control. Practical assessment. Criteria for degrees of control ${ }^{\mathbf{a}}$

Mild diabetes

\begin{tabular}{|c|c|c|c|c|}
\hline & \multicolumn{4}{|l|}{ Glucose } \\
\hline & \multicolumn{2}{|c|}{ Plasma mg/dl } & \multicolumn{2}{|l|}{ Urine } \\
\hline & Fasting & $\begin{array}{l}2 \mathrm{~h} \text { post- } \\
\text { prandial }\end{array}$ & Fasting & $\begin{array}{l}2 \mathrm{~h} \text { post- } \\
\text { prandial }\end{array}$ \\
\hline Ideal & $<110$ & $<110$ & $-v e$ & - ve \\
\hline Adequate & $\leq 130$ & $\leq 170$ & -ve & - ve or small \\
\hline Inadequate & $>130$ & $>170$ & $+v e^{b}$ & large \\
\hline
\end{tabular}

a Average of repeated observations under consistent conditions

b Small, moderate or large

sary and/or the prolonged avoidance of rapidly absorbable carbohydrates in the diet, may be reflected in an improved glucose tolerance test. The preparation for the initial and subsequent tests should be identical, with high carbohydrate intake for three days. The criteria for normality and for diabetes based on the oral glucose tolerance test are presently being reevaluated [32] but in the mildest diabetics normality should be aimed for.

An alternative to normal glucose tolerance testing is to provide the patient with a standard meal of known composition (such as a 100 gram carbohydrate breakfast) followed by two and/or three hour postprandial plasma glucose determination(s). This has the advantage of greater similarity (and therefore relevance) to everyday dietary "test-loads" as well as the need for fewer blood samples. For a normal response the plasma value at two hours should be below the upper limit of the normal range for the glucose method used (usually $110 \mathrm{mg} / \mathrm{dl}$ ) and at three hours it should be well within the normal fasting range (at or below $100 \mathrm{mg} / \mathrm{dl}$ ). If the therapy has achieved the desired weight reduction, it may be reasonable to expect that normal or near-normal values may be obtained if the patient is free from stress and intercurrent health problems.

Postprandial plasma glucose measurements can only be interpreted adequately if the composition (carbohydrate, fat and protein) and energy content of the food eaten in the preceding meal and the elapsed time since the start and end of the meal are known (Table 3). An ideal (normal) value at two hours would be $110 \mathrm{mg} / \mathrm{dl}$ or less. A value indicating control in the adequate range would have to be at or below $170 \mathrm{mg} / \mathrm{dl}$. A higher value two hours postprandially would usually be associated with glycosuria and should be considered as inadequate control of mild diabetes. 
Table 4. Diabetic control. Practical assessment. Criteria for degrees of control ${ }^{\mathrm{a}}$

Severe diabetes

\begin{tabular}{|c|c|c|c|c|}
\hline & \multicolumn{4}{|l|}{ Glucose } \\
\hline & \multicolumn{2}{|c|}{ Plasma mg/dl } & \multicolumn{2}{|l|}{ Urine $^{c}$} \\
\hline & Fasting & Preprandial $^{\mathrm{b}}$ & Fasting & Preprandial ${ }^{b}$ \\
\hline Ideal & $\leq 110$ & $\leq 110$ & - ve & $-\mathrm{ve}$ \\
\hline Adequate & $\leq 170$ & $\leq 170$ & -ve & -ve or small \\
\hline Inadequate & $>170$ & $>170$ & $+v e^{d}$ & large \\
\hline
\end{tabular}

a Average of repeated observations under consistent conditions

b As long after the preceding major meal as possible

c Quantitative: Ideal = no glycosuria (see text)

Adequate $\leq 5$ per cent of carbohydrate calories ingested

Inadequate $\supseteq 10$ per cent of carbohydrate calories ingested

d Small, moderate or large

Fasting plasma glucose determinations are useful to indicate whether these remain in the normal range, or if they have returned to that range. The timing of fasting values 10 to 16 hours after the last meal is most convenient. Unlike postprandial plasma glucose values, there need be less concern about precise timing in relation to the preceding meal and about the contents of the preceding meal.

Because paradoxically the mildest diabetics are the most likely to be tested rigorously with glucose tolerance tests, it is well to take advantage of such tests to determine the patient's blood-urine glucose relationship. By obtaining a baseline and subsequent hourly urine glucose measurements together with the blood samples during glucose tolerance testing, useful clues about the patient's "threshold" for glucose may be obtained.

It is generally recommended that urine testing for glucose be done as long after meals as possible and on second-voided specimens. This may not be ideal in mild diabetes, even though such testing may be best for insulin treated severe diabetic patients. Urine glucose testing two hours after meals is more likely to reveal glycosuria than at four or five hours (Table 3). The effectiveness of the therapy with diet, exercise or other measures may also best become evident at the two hour postprandial time point, if any previously observed glycosuria disappears. This should be seen with ideal and even with "just adequate" diabetic control.

Quantitative urine glucose tests on samples collected over 24-hourly periods may be used to give an estimate of the diabetic control. Whether the collection is done in the home or in the hospital, providing that it is a complete collection, a more complete assessment is feasible and day-to-day comparisons are also possible with no great inconvenience (see below for details).

Urinary ketone body testing is of little or no value to the non-insulin-treated diabetic. Tests to rule out hyperlipidaemia and serum protein abnormalities will indicate whether any follow-up is warranted on their behalf.

$\mathrm{Hb} \mathrm{AI}_{c}$, where available, should be a good method of testing for the degree of diabetic control over extended time periods $(21,23)$. This method could obviate the need for quantitative tests on urine collections with the greater convenience of just one blood sample and potentially greater precision.

Investigational Approach. However mild the impairment of carbohydrate tolerance may be, the documentation needs to be complete and with attention to the well known variations in glucose tolerance when glucose tolerance tests are repeated (33).

In addition to carbohydrate tolerance, lipid, protein and hormonal status should also be defined. Histocompatibility typing of mildly diabetic patients, who are the subjects of clinical investigations, is especially appropriate. With mild diabetes often being of relatively short duration it is desirable to seek for clues as to its ultimate type [6].

\section{Severe Diabetes}

Practical Approach. In patients who virtually or totally lack insulin, glucose tolerance testing is inappropriate. Fasting and postprandial tests of plasma glucose concentrations (after meals as provided in their individually prescribed diet) are appropriate and helpful.

In severely diabetic patients (who, for reasons of severe insulin deficiency or other reasons, are often difficult to manage) more moderate goals of therapy have to be accepted. The goal for the fasting plasma glucose value may be set as the (relatively) nearest to the normal range, because it follows the longest time period without eating. However, the avoidance of nocturnal hypoglycaemia in insulin-treated diabetics dictates caution in aiming for fasting euglycaemia. The individually determined "best compromise" results from these considerations [4].

Similar considerations apply to the setting of standards for tests just prior to other meals as well as breakfast (Table 4).

In contrast to mild diabetics in whom at two hours after meals a decrease of glycaemia to preprandial levels is sought, in severe diabetics at that time the postprandial peak of glycaemia is likely to be found 
[34]. In severe diabetic patients with individually selected insulin type and dosage, the nearest to normal glycaemia may be aimed for by four to six hours after the last meal. Avoidance of hypoglycaemia again imposes cautionary limits. Additional limiting factors are the patient's ability to respond adequately to insulin, especially during the forenoon, as well as dietary regimes, which provide between-meal feedings. With the latter, the time span between feedings is often reduced to two or three hours. Under these conditions the practical goal for preprandial glycaemia tends to be at or below $170 \mathrm{mg} / \mathrm{dl}$ (Table 4).

Recently much more rigorous criteria for glycaemic control have been made possible by the introduction of home blood glucose monitoring. Multiple samples may be checked using paper strips with the appropriate meter $[35,36]$. Acceptability to patients has been surprisingly good and the method is proving particularly useful in the management of labile and pregnant diabetics.

In clinical practice many of the problems concerning semiquantitative urine glucose testing may be avoided by decreasing the attention paid to the precision of the results. The information these tests provide indicates, (at best) whether there is glycosuria and if so, whether it is small ( $500 \mathrm{mg} / \mathrm{dl}$ or less), moderate $(1500 \mathrm{mg} / \mathrm{dl}$ or less) or large $(2000 \mathrm{mg} / \mathrm{dl}$ or more) $[10,11]$. Accordingly, if a method accomplishes such grading for a patient and if the recommendations for changes in therapy are appropriate, all that can be expected of semiquantitative tests may in fact, be used. With excessive attention to precision in fine shading of the degrees of urine glucose concentrations, which are not likely to relate dependably to plasma glucose concentrations (10), more harm than good may be caused.

A further practical rationalisation of the use of urine testing, to provide realistically optimal information about diabetic control, led us to the inclusion of semiquantitative tests for urinary ketone bodies in a useful seven-grade scale of assessment of diabetic control (Figure 2). In addition to the grades of "no glycosuria", "small", "moderate" or "large glycosuria" the testing, but (only) at the large glycosuria level for ketonuria as "none", "small", "moderate" and "large" in amount consistently improved the assessment of metabolic control (compared with consideration of degrees of glycosuria only). This was observed during studies of diabetic control carried out by continuous blood glucose monitoring and simultaneous urinary measurements $[10,16]$.

With quantitative urine glucose analysis the criteria recommended for evaluation of diabetic control commonly include relating the urine glucose content to the carbohydrate (or total caloric) content of

\section{DIABETIC CONTROL}

URINE TEST

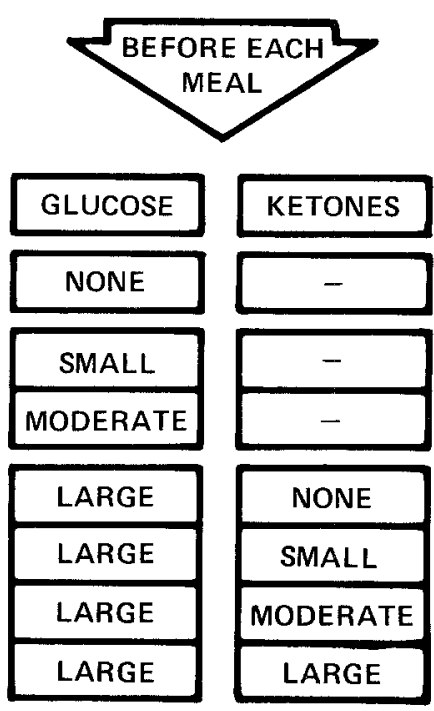

Fig. 2. Seven grade scale for semiquantitative urine glucose and ketone body tests. For each test negative and three grades of positive results are juxtaposed so that ketone bodies are measured only when large glycosuria is found

the diet. Thus $5 \%$ or less of the carbohydrate calories ingested appearing in the urine per 24 hours, is commonly regarded as adequate and $10 \%$ or more as inadequate diabetic control. Using sufficiently refined methods [10] it has been found that normal subjects may excrete urine containing 5 to $14 \mathrm{mg} / \mathrm{dl}$ glucose, especially postprandially. Obviously therefore, it is not realistic to aim for total absence of glycosuria, if a sufficiently sensitive analytic method is used.

$\mathrm{Hb} \mathrm{AI}_{\mathrm{c}}$ determinations will provide an excellent laboratory means for assessing diabetic control, as soon as this determination is sufficiently widely available. It must be kept in mind however, that this measurement is an indicator of the adequacy of diabetic control over long time periods of weeks and months [21-24]. Accordingly, it must not be substituted for the information needed to assess either the hour-to-hour changes in control or the day-to-day needs for therapeutic changes. The danger may be real, that the novelty and convenience of this good way of assessing long-term metabolic control may encourage physicians and patients alike to abandon the conventional techniques for monitoring diabetic control. Such exclusive reliance on $\mathrm{Hb} \mathrm{AI}$ measurements (at monthly intervals, for instance) could have disastrous consequences for severe and labile diabetic patients. However well the appropriate range for $\mathrm{Hb} \mathrm{AI}_{\mathrm{c}}$ may be selected and even attained month- 


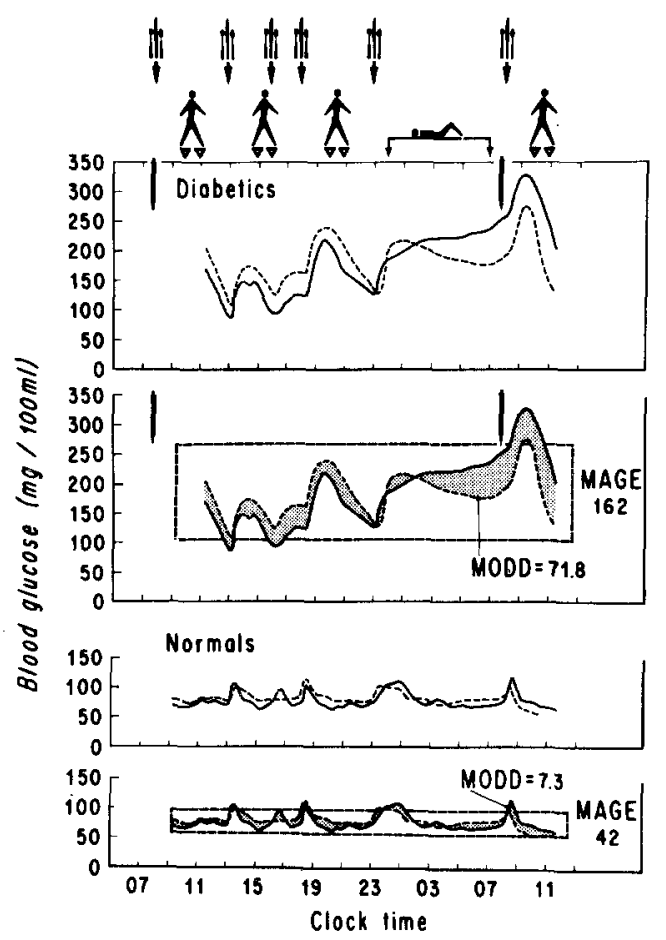

Fig. 3. Averaged blood glucose curves (continuously recorded) in six insulin dependent diabetic patients and in three normal volunteers. Symbols: fork-knife-spoon $=$ meals; walking figure $=$ exercise; reclining figure $=$ sleep; syringe and needle $=$ insulin injection. The glucose curve of the first 24 hours is a solid line and that of the second 24 hours is an interrupted line. Below each set of curves are shown the variability measurements for the same set of curves. The absolute difference between the first and second $24 \mathrm{~h}$ is the mean of daily differences of paired blood glucose values (MODD: shaded area). The mean amplitude of glycaemic excursions (MAGE) averaged for each group is shown bracketing the glucose curves. The values shown for MODD and MAGE are in $\mathrm{mg}$ per $/ 100 \mathrm{ml}$

by-month, this by itself will do little to help with avoiding hypoglycaemic episodes and periods of excessive postprandial hyperglycaemia. There is even more danger in that the necessary therapeutic changes for intercurrent illnesses of the severe diabetic may be unduly delayed or omitted, if excessive reliance on $\mathrm{Hb} \mathrm{AI}_{\mathrm{c}}$ monitoring of diabetic control is allowed to develop.

Attention to lipid, protein and hormonal aspects of the laboratory assessment should be considered early in the course of therapy. For instance, it is well to have made a diagnosis of hyperlipidaemia early in the formulation of diagnostic and therapeutic plans. Local and regional laboratory capabilities may determine, whether it will be possible to document and quantify a severe diabetic patient's residual insulin secretory ability or plasma glucagon response to hypoglycaemia (Figure 1). When possible, these should be tested, especially in patients whose diabetic management is difficult.
Investigational Approach. Complete documentation of severity, and variability of the response to treatment, are especially important in severe diabetic patients undergoing clinical investigations. The tests of insulinogenic reserve and endogenous glucagon response to hypoglycaemia described as optional in the practical-clinical setting are mandatory in the research setting for correct categorisation and adequate characterisation.

If C-peptide assays are available, the test with intravenous glucagon or with oral glucose or with a standard meal of carbohydrate and protein make it possible to determine whether the patient has any endogenous insulin secretory reserve $[3,5]$. Even if immunoreactive glucagon assays are available, testing the patient's ability to mobilise endogenous glucagon during hypoglycaemia may be difficult and even hazardous unless the insulin is infused slowly $(0.1 \mathrm{unit} / \mathrm{kg} / \mathrm{hour}$, Figure 1) and the plasma glucose monitored at the bedside [27]. This method overcomes the problems with intravenous injection of insulin which in severe diabetics may fail to produce hypoglycaemia if the initial glycaemia is too high or may produce excessive hypoglycaemia if the initial glycaemia is too low.

For assessment of blood glucose control prior to continuous in vivo glucose recordings, the "M" value of Schlichtkrull [37] was calculated on the basis of five to seven daily time-points over several successive days. It was the abundance of data generated by continuous recording which prompted the calculation of additional derived indices of blood glucose control $[2,38]$. Unlike the " $M$ " value, which is a weighted average of the glucose values (with due attention to the greater importance of lower-than-normal than to higher-than-normal values [37], the newer indices are based on separate analyses of within-day and between-day blood glucose fluctuations. Accordingly, the mean amplitude of glycaemic excursions (MAGE) averages the extent of each of the major glucose fluctuations from highest to lowest points during a 24 to 48 hour period [38] (Figure 3). The result is the average extent of glucose swings, a numerical expression for the within-day glucose fluctations. The mean of daily differences (MODD) averages the differences between glucose levels at the same time-points on successive days [2]. MODD is a numerical expression for between-day glucose fluctuations. Only continuously recorded data provide the precise highest and lowest value for each fluctuation, as well as truly representative data of changes from day-to-day [34] (Figure 3). It is possible, however, to find a plasma glucose value which correlates highly and positively with MAGE by sampling 80 minutes after breakfast [34]. Lev-Ran recently confirmed that 
a limited number of identically timed plasma glucose values on successive days can be used to document the between-day glucose variability of diabetics on consistent therapeutic regimens [30].

What is the value of such variability measurements? Glucose variability measurements identify characteristics of patients, which are otherwise not evident [2]. Compared to normal non-diabetic subjects, some diabetics on a standard therapeutic regimen have both high within-day and between-day glucose fluctuations (Figure 3), but others have mainly either high within-day or high between-day glucose fluctuations - not both. The within-day and betweenday glucose variabilities are partly independent one of another [2]. These determinations aid in research on brittle diabetes and also help practically in designing individually optimal therapeutic programmes for difficult-to-treat diabetic patients [39]. For the former purpose, glucose variability measurements have found application by researchers working on the "artificial pancreas" or on substitutes for it $[1,40$, 41]. Another application of glucose variability measurements has been the use of paired glucose values on successive days to reveal the apparently spontaneous effect of the latter half of pregnancy in stabilising brittle diabetes [42]. For adequate assessment of a patient with a management problem, it is necessary to gauge the extent and variability of glucose fluctuations. This is feasible even with intermittent but precisely timed multiple blood and urine samplings on successive days $[27,30,34]$. Isolated observations just on one day, or just at one time of day even if on successive days, is likely to give an incomplete and often misleading impression except in quite stable diabetics.

A useful refinement of quantitative glucose analysis on fractional urine collections, is the ability to compare glycosuria from one segment to another of the same 24 hour period. Thus day and night segments can be compared. If collections are made from before one meal until the next, shorter segments of the diurnal cycle may be compared. This is, in fact a within-day urine glucose variability measurement. By comparing the same diurnal segments from one day to the next, a between-day urine glucose variability measurement may be done. Thus urine glucose monitoring (but only with quantitative analysis) is appropriate for providing information about the amount of glycosuria and its variability. Although it is not possible to estimate the mean glycaemia from the amount of glycosuria, despite their generally high and positive interrelationship [10], urine glucose monitoring is well suited to "fill the gaps" of information when blood glucose is intermittently determined $[27,34]$.
With regard to the goals of therapy, research on severely diabetic patients may have as its aim the normalisation of some or all aspects of the demonstrable abnormalities. This aim then is a clear requirement to invoke most, if not all of the methods already cited to document what is amiss with the patient and to what extent the experimental therapy succeeded [4]. If the research aims do not involve metabolic normalisation, then the goals of therapy have to be the best compromise as described under the practical approach for severely diabetic patients [39]. In the latter instance only the greater completeness of documentation will distinguish the investigational from the practical approach as to the degree of diabetic control that is desirable, feasible and documentable.

\section{References}

1. Marliss, E. B., Murray, F. T., Stokes, E. F., Zinman, B., Makhooda, A. F., Denoga, A., Leibel, B. C., Albisser, A. M.: Normalization of glycemia in diabetics during meals with insulin and glucagon delivery by the artificial pancreas. Diabetes 26, 663-672 (1977)

2. Molnar, G. D., Taylor, W. F., Ho, M. M.: Day-to-day variation of continuously monitored glycemia: a further measure of diabetic instability. Diabetologia 8, 342-348 (1972)

3. Kuzuya, H., Blix, P.M., Horwitz, D. L., Steiner, D. F., Rubenstein, A. H.: Determination of free and total insulin and C-peptide in insulin-treated diabetics. Diabetes 26, 22-29 (1977)

4. Molnar, G. D.: Metabolic derangements of diabetes - The challenge of normalization. In: Diabetes. Bajaj, J.S. (Ed.), pp. 456-463. ICS 413. Amsterdam: Excerpta Medica 1977

5. Rubenstein, A. H., Kuzuya, H., Horwitz, D. L.: Clinical significance of circulating $\mathrm{C}$-peptide in diabetes mellitus and hypoglycemic disorders. Arch. Intern. Med. 137, 625-632 (1977)

6. Nerup, J., Platz, P., Ryder, L. P., Thomsen, M., Svejgaard, A.: HLA, islet cell antibodies and types of diabetes mellitus. Diabetes 27, (Suppl. 1), 247-250 (1978)

7. Molnar, G. W., Read, R. C.: Hypoglycemia and body temperature. J.A.M.A. 227, 916-921 (1974)

8. Neijadlik, D. C., Dube, A. H., Adamko, S. M.: Glucose measurements and clinical correlations. J.A.M.A. 224, 1734-1736 (1973)

9. Lewis, J. G., Stephens, P. J.: Tear glucose in diabetics. Pr. J. Ophthalmol. 42, 754-758 (1958)

10. Service, F. J., Molnar, G. D., Taylor, W. R.: Urine glucose analyses during continuous blood glucose monitoring. J.A.M.A. 222, 294-298 (1972)

11. James, R. C., Chase, G. R.: Evaluation of some commonly used semiquantitative methods for urinary glucose and ketone determinations. Diabetes 23, 474-479 (1974)

12. Shafrir, E.: Hyperlipidemia in diabetes. In: Diabetes Mellitus. Sussman, K. E., Metz, R.J.S. (Eds.), Vol. 4, pp. 221-228. New York: American Diabetes Association 1975

13. Sherwin, R. S., Hendler, R. J., Felig, P.: Effect of diabetes mellitus and insulin on the turnover and metabolic response to ketones in man. Diabetes 25, 776-784 (1976)

14. McGarry, J.D., Foster, D.W.: Hormonal control of 
ketogenesis - Biochemical considerations. Arch. Intern. Med. 137, 495-501 (1977)

15. Barnes, A. J., Bloom, S. R., Alberti, K. G. M. M., Smythe, P., Alford, F. P., Chisholm, D. J.: Ketoacidosis in pancreatectomized man. N. Engl. J. Med. 296, 1250-1253 (1977)

16. Thomas, S. K., Taylor, W.F., Molnar, G. D.: Continuous blood glucose analysis in ambulatory fed subjects. III. Nitrogen balance and urinary ketone bodies related to other measurements in the characterization of unstable diabetes. Mayo Clin. Proc. 49, 28-33 (1974)

17. Felig, P., Wahren, J., Sherwin, R., Palaiologos, G.: Amino acid and protein metabolism in diabetes mellitus. Arch. Intern. Med. 137, 507-513 (1977)

18. Mogensen, C. E.: Renal function changes in diabetes. Diabetes 25 (Suppl. 2), 872-879 (1976)

19. Parving, H.H., Noer, I., Deckert, T., Evrin, P.-E., Nielsen, S. L., Lynsgøe, J., Mogensen, S. E., Rørth, M., Svendsen, P. Aa., Trap-Jensen, J., Lassen, N. A.: The effect of metabolic regulation on microvascular permeability to small and large molecules in short-term diabetes. Diabetologia 12, 161-166 (1976)

20. Koenig, R. J., Peterson, C. M., Kilo, C., Cerami, A., Williamson, J. R.: Hemoglobin $\mathrm{AI}_{\mathrm{c}}$ as an indicator of the degree of glucose intolerance in diabetes. Diabetes 25, 230-232 (1976)

21. Paulsen, E. P., Koury, M.: Hemoglobin $\mathrm{AI}_{\mathrm{c}}$ levels in insulindependant and -independant diabetes mellitus. Diabetes 25 (Suppl. 2), 890-896 (1976)

22. Peterson, C. M., Koenig, R. J., Jones, R. L., Saudek, C. D., Cerami, A.: Correlation of serum triglyceride levels and hemoglobin $\mathrm{AI}_{\mathrm{c}}$ concentration in diabetes mellitus. Diabetes 26, 507-509 (1977)

23. Graf, R. J., Halter, J. B., Porte, D.: Glycosylated hemoglobin in normal subjects and subjects with maturity-onset diabetes. Diabetes 27, 834-839 (1978)

24. Gonen, B., Rubenstein, A. H.: Haemoglobin AI and diabetes mellitus. Diabetologia 15, 1-8 (1978)

25. Spitz, I. M., Rubenstein, A. H., Bersohn, I., Wright, A. D., Lowy, C.: Urine insulin and renal disease. J. Lab. Clin. Med. 75, 990-1005 (1970)

26. Horwitz, D. L., Rubenstein, A. H., Katz, A. I.: Quantitation of human pancreatic beta-cell function by immunoassay of Cpeptide in urine. Diabetes 26, 30-35 (1977)

27. Reynolds, C., Molnar, G. D., Horwitz, D. L., Rubenstein, A. H., Taylor, W. F., Jiang, H.: Abnormalities of endogenous glucagon and insulin in unstable diabetes. Diabetes 26, 36-45 (1977)

28. Palumbo, P. J., Molnar, G. D., Taylor, W. F., Moxness, K. E., Tauxe, W. N.: Insulin antibody binding in diabetes mellitus and factitious hypoglycemia. Mayo Clin. Proc. 44, 725-737 (1969)
29. Dixon, K., Exon, P. D., Malins, J. M.: Insulin antibodies and the control of diabetes. J. Med. 44, 543-553 (1975)

30. Lev-Ran, A.: Clinical observations on brittle diabetes. Arch. Intern. Med. 138, 372-376 (1978)

31. Bar, R. S., Roth, J.: Insulin receptor status in diseased state of man. Arch. Intern. Med. 137, 474-481 (1977)

32. Keen, H., Jarrett, R. J., Alberti, K. G. M. M.: Diabetes mellitus: a new look at diagnostic criteria. Diabetologia 16, 283-285 (1979)

33. Wilkerson, H. L. C.: Diagnosis, oral glucose tolerance tests In: Diabetes mellitus. Danowski, T.S. (Ed.), Vol. I, pp. 31-34. New York: American Diabetes Association 1964

34. Molnar, G. D., Taylor, W. F., Langworthy, A.: On measuring the adequacy of diabetes regulation: comparison of continuously monitored blood glucose patterns with values at selected time points. Diabetologia 10, 139-143 (1974)

35. Sonksen, P. H., Judd, S. L., Lowy, C.: Home monitoring of blood glucose. Lancet 1978 I, 729-732

36. Walford, S., Gale, E. A. M., Allison, S. P., Tattersall, R. B.: Self monitoring of blood glucose. Lancet 1978 I, 732-735

37. Schlichtkrull, J., Munck, O., Jersild, M.: The M-value, an index of blood sugar control in diabetics. Acta Med. Scand. 177, 95 (1965)

38. Service, F. J., Molnar, G. D., Rosevear, J. W., Ackerman, E., Gatewood, L. C., Taylor, W. F.: Mean amplitude of glycemic excursions, a measure of diabetic instability. Diabetes 19, 644-655 (1970)

39. Molnar, G. D.: Unstable diabetes: concepts of its nature and treatment based on continuous blood glucose monitoring studies. In: Diabetes. Malaisse, W.J., Pirart, J. (Eds.), pp. 546-559, ICS 312. Amsterdam: Excerpta Medica 1974

40. Mirouze, J., Collard, F., Selam, J. L., Pham, T. C.: Continuous blood glucose monitoring in insulin-treated diabetes. Horm. Metab. Res. (Suppl.) 7, 77-86 (1977)

41. Pfeiffer, E. F., Beischer, W., Kerner, W.: The artificial endocrine pancreas in clinical research. Horm. Metab. Res. (Suppl.) 7, 95-114 (1977)

42. Lev-Ran, A., Goldman, J. A.: Brittle diabetes in pregnancy. Diabetes 26, 926-930 (1977)

Received: February 14, 1979

Dr. G. D. Molnar

The University of Alberta

Department of Medicine

8-121 Clinical Sciences Building

Edmonton, Alberta

Canada T6G 2G3 